\title{
Factores psicosociales asociados al consumo de sustancias psicoactivas en adolescentes de Pereira, Colombia
}

\author{
Psychosocial factors associated with the use of psychoactive substances in children and adolescents from Pereira, \\ Colombia
}

\author{
Ángela María Álvarez-López ${ }^{1,2}$ orcid.org/0000-0001-6740-0322 \\ Natalia Jeaneth Carmona-Valencia1, ${ }^{1 *}$ orcid.org/0000-0002-0129-9011 \\ Ángela Liceth Pérez-Rendón ${ }^{3,4}$ orcid.org/0000-0002-2363-8782 \\ Adriana Jaramillo-Roa ${ }^{12}$ orcid.org/0000-0002-3385-4283
}

1 Programa de Terapia Respiratoria, Fundación Universitaria del Área Andina. Pereira, Colombia
2 Grupo de investigación ZIPATEFI, Fundación Universitaria del Área Andina. Pereira, Colombia
3 Departamento de Ciencias Básicas, Fundación Universitaria del Área Andina. Pereira, Colombia
4 Grupo de investigación GIEE, Fundación Universitaria del Área Andina. Pereira, Colombia

Fecha de recepción: Marzo 8 - 2019

Fecha de revisión: Julio 26 - 2019

Fecha de aceptación: Agosto 28 - 2020

Álvarez-López AM, Carmona-Valencia NJ, Pérez-Rendón AL, Jaramillo-Roa A. Factores psicosociales asociados al consumo de sustancias psicoactivas en adolescentes de Pereira, Colombia. Univ. Salud. 2020;22(3):213-222. DOI: https://doi.org/10.22267/rus.202203.193

\section{Resumen}

Introducción: El consumo de sustancias psicoactivas es un problema de salud pública con alta prevalencia en los adolescentes, situación que influye en el desarrollo individual y social en la adultez. Objetivo: Determinar los factores psicosociales relacionados al consumo de sustancias psicoactivas en adolescentes escolarizados de básica secundaria y educación media de una Institución Educativa pública del área urbana de Pereira. Materiales y métodos: Estudio descriptivo correlacional con 235 estudiantes de un colegio público, entre 10 y 19 años de edad, quienes completaron una encuesta anónima que indagaba la prevalencia y los factores psicosociales asociados al consumo. Resultados: Se encontró que la adecuada supervisión de los padres es un factor protector frente al consumo de sustancias psicoactivas $(p<0,05)(O R<1)$, los escolares tienen 6,5 veces más posibilidades de consumir cuando se relacionan con amigos que consumen [OR=6,555 IC95\% (3,147-13,653)], los que tienen familiares que consumen, tienen 2,2 veces más posibilidades de consumir estas sustancias [OR=2,231 IC95\% (1,292-3,852)]. Conclusiones: El consumo de sustancias psicoactivas muestra una elevada prevalencia en los escolares encuestados y los principales factores relacionados son cursar grados superiores y tener familiares o amigos que consumen sustancias psicoactivas.

Palabras clave: Drogas Ilícitas; factores de riesgo; estudiantes; condiciones sociales. (Fuente: DeCS, Bireme).

\begin{abstract}
Introduction: The use of psychoactive substances is a public health problem with a high prevalence in adolescents, which also affects the individual and social development of adults. Objective: To determine the psychosocial factors related to the use of psychoactive substances in children and adolescents of a public school from Pereira (Colombia). Materials and methods: A descriptive correlational study was conducted on 235 students of a public school, aged between 10 and 19 years, who completed an anonymous survey to examine the prevalence and psychosocial factors associated with the use of psychoactive substances. Results: Adequate parental supervision is a protective factor against psychoactive drug use $(p<0.05)(O R<1)$. Schoolchildren are 6.5 times more likely to engage in drug use when they interact with psychoactive-drug user friends [OR=6.555 95\%CI $(3.147$ 13.653)]. Those with family members who engage in this drug use have a 2.2 times higher possibility to use psychoactive substances [OR=2.231 95\%CI (1.292-3.852)]. Conclusions: The use of psychoactive substances shows a high prevalence in the participating schoolchildren and is mainly related to factors such as being in higher grades in their school and having either a family member or a friend who uses psychoactive substances.
\end{abstract}

Keywords: Street drugs; risk factors; students; social conditions. (Source: DeCS, Bireme).

\footnotetext{
*Autor de correspondencia

Natalia Jeaneth Carmona Valencia

e-mail: ncarmona8@areandina.edu.co
} 


\section{Introducción}

La adolescencia es una etapa de cambios físicos y psicosociales, en la que se adoptan conductas de riesgo dentro de una difícil búsqueda de equilibrio entre la dependencia familiar y la autonomía personal, es considerada como el período en el cual el individuo adquiere la capacidad de reproducirse, transita desde los patrones psicológicos de la niñez a los de la vida adulta. Transcurre entre los 10 y 19 años de edad y representa aproximadamente una sexta parte de la población mundial (1.200 millones de personas) $)^{(1)}$.

La adolescencia se caracteriza por el esfuerzo para independizarse de los padres y demás adultos, estrechar la amistad con otros jóvenes, experimentar situaciones y estilos de vida que a veces implica asumir mayores riesgos, tomar decisiones, aceptar compromisos y aprovechar oportunidades que podrían conducir a resultados inciertos(2). El consumo de sustancias psicoactivas entre los adolescentes, se está convirtiendo en una vía para escapar de situaciones en las que suelen sentirse impotentes de modificar(3).

En el mundo el consumo de sustancias psicoactivas es considerado un problema de salud pública; a pesar de los esfuerzos por prevenir o controlar este flagelo en la adolescencia(4), según el informe de la Oficina de las Naciones Unidas contra la Droga y el Delito, del año 2017, un cuarto de billón de personas, o cerca del 5\% de la población entre los 15 y los 64 años, consumió drogas por lo menos en una ocasión en 2015(5).

En Colombia, de acuerdo a lo dispuesto en las leyes 124 de 1994 y 1335 de 2009, se prohíbe el consumo de tabaco y alcohol en menores de 18 años, por tal motivo estas dos sustancias son consideradas ilícitas en los menores de edad(6,7). De acuerdo a tres estudios realizados en Colombia en población adolescente entre los años 2004 y 2016, se ha logrado disminuir la prevalencia en el consumo de tabaco, pero las demás sustancias psicoactivas permanecen estables o continúan aumentado(8-10).

Según el estudio del año 2016, el 70,7\% de los estudiantes usaron una sustancia psicoactiva alguna vez en la vida, el 61,3\% la usó en el último año y un $39,1 \%$ en el último mes, siendo importante recalcar que el $15,9 \%$ de los estudiantes declararon haber consumido por lo menos una sustancia ilícita alguna vez en la vida. En esta población, la segunda sustancia de mayor uso es el tabaco, pero con cifras muy inferiores a las descritas para el alcohol; en tercer lugar, se ubica la marihuana y luego los estimulantes $^{(10)}$.

Con respecto a si los jóvenes habían consumido alguna vez en su vida sustancias psicoactivas ilícitas, el departamento de Risaralda supera los datos nacionales en 10,1 puntos porcentuales, ocupando el tercer lugar en el país, con un consumo de $26,1 \%$, encontrándose sobre la media nacional en relación al uso de alcohol, marihuana, cocaína, éxtasis, dick, Popper, alucinógenos y bebidas energizantes. En este departamento las sustancias psicoactivas que se encuentran bajo la media nacional son el tabaco, el bazuco, el LSD (Dietilamida del Ácido Lisérgico), pegamentos, solventes y pinturas ${ }^{(10)}$

El consumo de sustancias psicoactivas es un fenómeno complejo que no depende solamente de la persona que consume, es un proceso donde intervienen la familia, la sociedad y el entorno, y debe ser afrontado desde un enfoque integral. Para la comprensión de este fenómeno es necesario identificar y articular tres escenarios: la familia, la comunidad y la escuela, cuyas interacciones determinan la exposición al riesgo de consumo de sustancias psicoactivas(2-6,11).

La organización y la dinámica familiar ofrecen al joven un contexto de desarrollo psicosocial enmarcado en valores, principios y comportamientos que determinan su futuro. La familia y la escuela comparten un escenario: la comunidad. Las interacciones con la comunidad, algunas derivadas de las relaciones escolares y otras surgidas durante la convivencia con los vecinos, marcan unos lineamientos de vulnerabilidad o factores protectores frente al inicio o el mantenimiento de un determinado consumo(11).

Investigaciones sugieren que el consumo de sustancias psicoactivas puede estar relacionado con el tipo de familia(12-15), nivel de escolaridad de los padres(16) y/o nivel de supervisión en la vida del adolescente(10,12,15-18). Teniendo en cuenta la influencia de familiares y amigos consumidores(19-21), muchos adolescentes empiezan el consumo motivados por el comportamiento de sus pares y en busca de una aceptación positiva directa, obtenida de 
los efectos placenteros del consumo. En ocasiones la presión de grupo, entendida como la exigencia que realizan los jóvenes a los integrantes del grupo al que pertenecen, puede dar más importancia a los aspectos grupales que a los individuales ${ }^{(22)}$.

A nivel mundial se reconoce la prevalencia(7-10,23-27) y los factores psicosociales que influyen en el consumo de sustancias psicoactivas(10,12-22,28), sin embargo, son pocos los estudios que sustentan esta problemática en de Pereira, por ello es importante determinar los factores psicosociales relacionados al consumo de sustancias psicoactivas en adolescentes escolarizados de básica secundaria y educación media de una Institución Educativa pública del área urbana de Pereira.

\section{Materiales y métodos}

Se realizó una investigación con diseño observacional, descriptivo, de corte trasversal con enfoque cuantitativo, en una Institución Educativa de la ciudad de Pereira. La población del estudio se constituyó con 391 adolescentes matriculados en la Institución Educativa; el tamaño de muestra se estableció con un nivel de confianza del $95 \%$ y un error del $4 \%$, con lo cual se estableció una muestra de 235 estudiantes.

El instrumento de recolección de datos fue elaborado por los investigadores con la finalidad de abarcar tres componentes:

1. Caracterización sociodemográfica, el cual consta preguntas referentes a la edad, grado escolar y género.

2. Consumo de sustancias psicoactivas lícitas e ilícitas basado en las encuestas nacionales de consumo de sustancias psicoactivas en adolescentes colombianos (8-10).

3. Factores relacionados al consumo, se indagó sobre aspectos como tipo de familia, escolaridad de los padres, consumo en familiares $y$ amigos e involucramiento parental.

Dicho instrumento fue revisado por tres profesionales, dos psicólogos clínicos y un investigador experto en sustancias psicoactivas para la validación del contenido; en relación a la forma, se modificó el cuestionario de acuerdo a las inconsistencias encontradas, posteriormente se aplicó la prueba de confiabilidad de alfa Cronbach obteniendo un valor de 0,81 .
El instrumento tipo encuesta auto diligenciada, se aplicó en las aulas de clase de la Institución Educativa, de forma individual, guiados por uno de los investigadores, el procedimiento tuvo una duración aproximada de 20 minutos.

La información se procesó en el paquete estadístico, SPSS v.20 (Statistical Pakage for the Social Science). En el análisis descriptivo se obtuvieron las medias, desviaciones o proporciones de las variables. En el análisis bivariado se analizó la relación entre las variables cualitativas a partir de la prueba Chicuadrado $\left(X^{2}\right)$, el nivel de significancia estadística se estableció en $(p<0,05)$. Finalmente, para determinar la asociación entre las variables padres involucrados y el consumo de los escolares de sustancias Psicoactivas se utilizó el índice de Odds Ratio (OR) con su intervalo de confianza del 95\% (IC95\%).

\section{Consideraciones éticas}

Para dar cumplimiento a la reglamentación sobre aspectos bioéticos se revisó la declaración de Singapur(29) y en Colombia la Resolución 008430 de 1993(30) de acuerdo a la cual debe contarse con aprobación de acudientes y adolescentes, los cuales fueron firmados a través de los consentimientos informados. Adicionalmente, se contó con el aval institucional. De acuerdo a la norma ISO 26000 de 2012(31), el equipo investigador asumió como responsabilidad social el acompañamiento a la institución en actividades dirigidas a intervenir los factores relacionados al consumo de sustancias psicoactivas, por medio de una jornada de sensibilización en compañía de entidades gubernamentales de la ciudad de Pereira.

La investigación fue aprobada por el comité de investigación de la Facultad de Salud de la Fundación Universitaria del Área Andina sede Pereira.

\section{Resultados}

\section{Características sociodemográficas}

En el estudio participaron 235 alumnos pertenecientes al estrato socioeconómico medio bajo, el 59,6\% pertenecían al género masculino y el $40,4 \%$ al femenino. Los participantes tenían edades entre $\operatorname{los} 10$ y 19 años con un promedio de $14,17 \pm 1,86$ años. 
La Tabla 1 evidencia los factores sociales de los adolescentes, donde se puede observar el grado de escolaridad de los participantes, seguido del núcleo familiar al que pertenecen y nivel de escolaridad de los padres, resaltando que el $51,5 \%$ de los familiares son consumidores y el $66,4 \%$ tienen amigos consumidores.

Tabla 1. Distribución de factores sociales de los adolescentes escolarizados en un colegio Público de la ciudad de Pereira, 2018

\begin{tabular}{|c|c|c|}
\hline Variable & $\mathbf{N}$ & $\%$ \\
\hline \multicolumn{3}{|l|}{ Grado escolar } \\
\hline Sexto & 45 & 19 \\
\hline Séptimo & 48 & 20,4 \\
\hline Octavo & 36 & 15,3 \\
\hline Noveno & 36 & 15,3 \\
\hline Decimo & 40 & 17 \\
\hline Once & 30 & 12,8 \\
\hline \multicolumn{3}{|l|}{ Tipo de familia } \\
\hline Nuclear & 71 & 30,2 \\
\hline Extendida & 97 & 41,3 \\
\hline Monoparental & 21 & 7 \\
\hline Ensamblada & 30 & 12,8 \\
\hline Homoparental & 2 & 0,9 \\
\hline De hecho & 1 & 0,4 \\
\hline No responde & 13 & 5,5 \\
\hline \multicolumn{3}{|l|}{ Familiares que consumen } \\
\hline $\mathrm{Si}$ & 121 & 51,5 \\
\hline No & 114 & 48,5 \\
\hline \multicolumn{3}{|l|}{ Amigos que consumen } \\
\hline$\overline{\mathrm{Si}}$ & 156 & 66,4 \\
\hline No & 79 & 33,6 \\
\hline \multicolumn{3}{|c|}{ Cuántas veces ocurre que sus padres no saben dónde está después que sale del colegio } \\
\hline Siempre & 212 & 90,2 \\
\hline Casi nunca & 23 & 9,8 \\
\hline \multicolumn{3}{|c|}{ Sus padres se fijan en los programas que usted ve en la televisión } \\
\hline $\mathrm{Si}$ & 151 & 64,2 \\
\hline No & 84 & 35,8 \\
\hline \multicolumn{3}{|c|}{ Sus padres están atentos de lo que usted hace en el colegio } \\
\hline Mucho & 194 & 82,6 \\
\hline Poco & 41 & 17,4 \\
\hline \multicolumn{3}{|c|}{ Durante los fines de semana, sus padres controlan la hora de llegada } \\
\hline $\mathrm{Si}$ & 208 & 88,5 \\
\hline No & 27 & 11,5 \\
\hline \multicolumn{3}{|c|}{ Al salir de casa sus padres le preguntan y/o esperan que diga a dónde va } \\
\hline $\mathrm{Si}$ & 214 & 91,2 \\
\hline No & 21 & 8,9 \\
\hline \multicolumn{3}{|l|}{ Días a la semana que se sientan a comer juntos } \\
\hline 4 o más días & 165 & 70,2 \\
\hline 3 o menos días & 70 & 29,8 \\
\hline \multicolumn{3}{|l|}{ Sus padres conocen a sus amigos más cercanos } \\
\hline Bastante & 157 & 66,8 \\
\hline Poco & 78 & 33,2 \\
\hline
\end{tabular}

Con respecto al nivel escolar de los padres se encontró que 49,7\% de los jóvenes afirmaron que sus madres cuentan con educación secundaria y el 14,4\% tiene un nivel de escolaridad de primaria. A su vez, se observó que el 31,9\% de los padres cuentan con el nivel de secundaria terminado y un $25,9 \%$ no tiene conocimiento sobre el nivel académico de su padre. El $6,38 \%$ de los estudiantes expresaron que sus madres 
y padres asistieron a alguna institución de educación superior.

\section{Prevalencia de consumo}

El 36,6\% de la población encuestada reportó haber consumido algún tipo de sustancia psicoactiva alguna vez en su vida, siendo un $58,4 \%$ del género masculino y un $41,6 \%$ del femenino.

Los escolares a partir del grado séptimo hasta el grado once han consumido en mayor proporción algún tipo de sustancias psicoactivas, evidenciado mayor consumo de alcohol, teniendo en cuenta que en los grados decimo y once se reporta el mayor porcentaje de consumo de varias sustancias (alcohol, marihuana, tabaco, Popper, ladies, pegante o fragancia) como se evidencia en la Figura 1.

En lo que respecta al ofrecimiento de sustancias psicoactivas, el $60 \%$ de los escolares encuestados manifestaron que nunca les han ofrecido comprar o probar algún tipo de sustancia psicoactiva; mientras que un 17,8\% de ellos indicaron que en los últimos 30 días se les ofreció comprar o probar dichas sustancias y el 8,08\% informaron que hace más de un año no les ofrecen este tipo sustancias.

$\mathrm{Al}$ indagar por los motivos de consumo, el 45,5\% de los escolares que han consumido alguna de las sustancias, indicaron que lo hicieron por curiosidad, un $13 \%$ por placer y en menor proporción indicaron

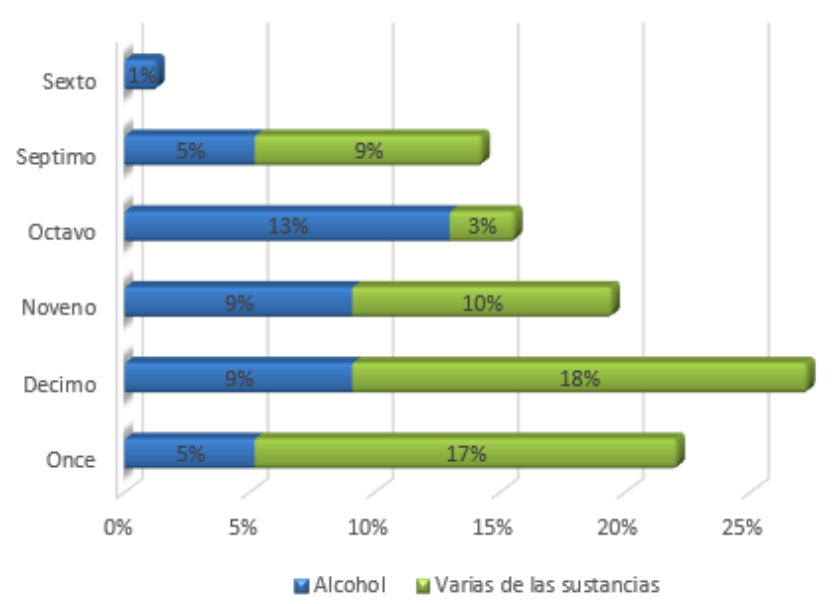

que el motivo de consumo fueron los problemas familiares con un 3,9\%, seguido por la presión de pares y baja autoestima con un 1,3\% cada uno de ellos, siendo importante resaltar que el $31,2 \%$ de los adolescentes manifestaron que su consumo se debe a varias de las opciones mencionadas dentro de la encuesta.

En cuanto a los lugares de consumo, el 18,2\% de los escolares refiere hacerlo en zonas recreativas, seguido del 15,6\% que consumen en fiestas, el 7,8\% en su propia casa y el 3,9\% en la casa de los amigos; en menor proporción el 1,3\% de los jóvenes han consumido en los alrededores o cerca al colegio; mientras que el $33,8 \%$ de los encuestados que han consumido alguna vez en la vida, indicaron que lo hacen en varios de los lugares mencionados y el $18,2 \%$ de ellos manifestó que actualmente no consume. También se indagó en los escolares que han consumido alguna vez en la vida, sobre la percepción que tienen con respecto a la accesibilidad y disponibilidad a las sustancias psicoactivas y el 76,6\% de ellos indicaron que es fácil acceder a ellas.

Con respecto a la oferta y consumo de sustancias psicoactivas y si se han consumido durante los últimos 12 meses, se encontró que el 70,6\% de los estudiantes afirman que al colegio algunos compañeros traen o pasan dichas sustancias, de estos $45,1 \%$ afirman no haber consumido en los últimos 12 meses.

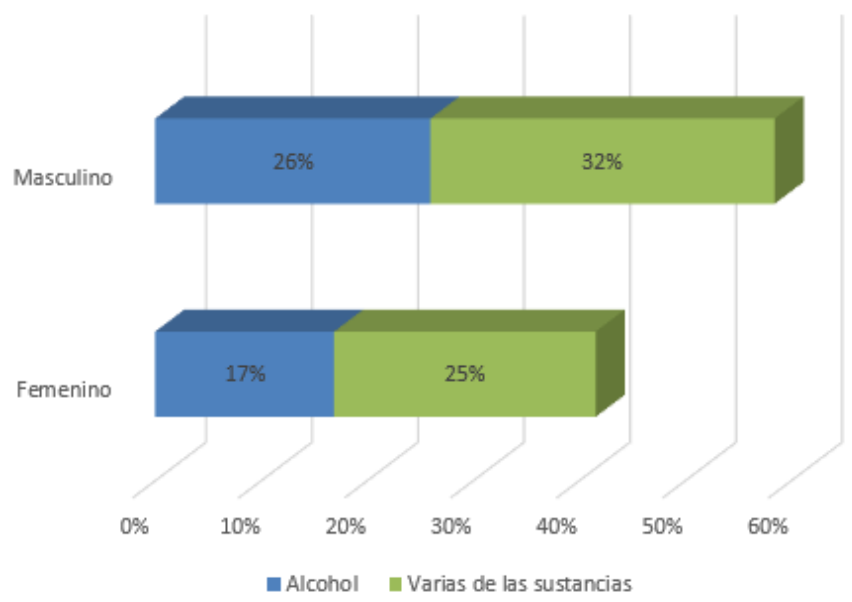

Figura 1. Consumo de sustancias psicoactivas con relación al grado escolar y el género

El $65,9 \%$ consideran que en los alrededores del plantel educativo sus compañeros adquieren este tipo de sustancias, de ellos el 45,5\% afirman no haber consumido en los últimos 12 meses y un $35,3 \%$ ha estado presente cuando se venden en el colegio o alrededores. Se encontró que el 59,5\% de los 
participantes afirman que han visto usar sustancias psicoactivas en el colegio, de ellos el $36,6 \%$ no han consumido durante los últimos 12 meses; existen diferencias significativas entre el consumo de los estudiantes con respecto al consumo durante los últimos 12 meses o último mes $p<0,05$, como se observa en la Tabla 2.

Tabla 2. Relación entre oferta y consumo en el entorno y consumo en los últimos 12 meses

\begin{tabular}{|c|c|c|c|}
\hline Oferta y consumo entorno & $\%$ & $X^{2}$ & P-value \\
\hline \multicolumn{4}{|c|}{$\begin{array}{l}\text { Considera usted que algunos estudiantes } \\
\text { pasan drogas entre ellos, dentro del colegio }\end{array}$} \\
\hline Sí & 70,6 & \multirow{2}{*}{2,931} & \multirow{2}{*}{0,231} \\
\hline No & 29,3 & & \\
\hline \multicolumn{4}{|c|}{$\begin{array}{l}\text { Considera usted que algunos estudiantes traen, prueban o } \\
\text { pasan drogas entre ellos, en los alrededores del colegio }\end{array}$} \\
\hline Sí & 65,9 & \multirow{2}{*}{0,700} & \multirow{2}{*}{0,705} \\
\hline No & 34,1 & & \\
\hline \multicolumn{4}{|c|}{$\begin{array}{l}\text { Ha visto alguna vez a un alumno vendiendo o pasando drogas } \\
\text { en el colegio o en los alrededores }\end{array}$} \\
\hline Sí & 35,3 & \multirow{2}{*}{0,091} & \multirow{2}{*}{0,956} \\
\hline No & 64,6 & & \\
\hline \multicolumn{4}{|c|}{ Ha visto usar drogas en el colegio o alrededores } \\
\hline Sí & 59,5 & \multirow{2}{*}{6,466} & \multirow{2}{*}{$0,039 *$} \\
\hline No & 40,4 & & \\
\hline
\end{tabular}

\section{Relación de los pares con el consumo de sustancias psicoactivas}

Al analizar si existe relación entre los pares y el consumo de sustancias psicoactivas de los estudiantes, se encontró que alrededor del $66,4 \%$ de los adolescentes afirman tener amigos que consumen sustancias psicoactivas, lo cual indica que tienen mayor posibilidad de consumir con una fuerza de asociación positiva, donde se observa que los escolares tienen 6,5 veces más posibilidades de consumir alucinógenos cuando se relacionan con amigos que consumen [OR=6,555 IC $95 \% \quad(3,147$ $13,653)]$. Lo mismo ocurre con la variable familiares que consumen $(p<0,05)$, donde se observa que el $51,5 \%$ de los escolares respondieron que tienen familiares que consumen, indicando que los estudiantes tienen 2,2 veces más posibilidades de consumir sustancias psicoactivas frente a los estudiantes que cuentan con un círculo familiar donde no consumen [OR=2,231 IC $95 \% \quad(1,292$ 3,852)].

Por otra parte, se encontró que existen diferencias significativas entre algunos ítems de la escala de padres involucrados con respecto a la pregunta sobre el consumo de cualquier sustancia psicoactiva alguna vez en la vida, como se observa en la Tabla 3.

Se resalta que no existen diferencias significativas entre el consumo y si los padres conocen sus amigos. El estudio también permitió establecer que, si lo padres están pendiente de sus hijos, saben dónde están los fines de semana, se fija en el tipo de programas que ven y están al tanto de los que hacen en el colegio, los escolares tienen menos probabilidad de consumir sustancias psicoactivas $(O R<1)$. Si los padres saben dónde están los adolescentes los fines de semana tienen 3,7 veces más posibilidades de evitar que sus hijos consuman sustancias psicoactivas, como se observa en la Tabla 3.

Tabla 3. Relación entre los padres involucrados y el consumo de sustancias Psicoactivas

\begin{tabular}{|c|c|c|c|c|c|}
\hline Escala de Padres involucrados & $X^{2}$ & G.L & P-value & Odd Ratio & IC $95 \%$ \\
\hline Los padres saben dónde están los fines de semana & 7,68 & 1 & $0,006^{*}$ & 0,27 & {$[0,109-0,663]$} \\
\hline Los padres se fijan en lo que ven en TV & 17,394 & 1 & $<0,0001^{*}$ & 0,30 & {$[0,170-0,522]$} \\
\hline Los padres prestan atención en lo que hacen en el colegio & 11,48 & 1 & $0,001^{*}$ & 0,29 & {$[0,146-0,589]$} \\
\hline Los padres controlan la hora de llegada & 1,237 & 1 & 0,266 & 0,58 & {$[0,260-1,305]$} \\
\hline Los padres preguntan para donde van & $<0,0001$ & 1 & 1,000 & 0,93 & {$[0,370-2,347]$} \\
\hline Días que comen en familia & 8,565 & 1 & $0,003^{*}$ & 0,41 & {$[0,231-0,729]$} \\
\hline Los padres conocen los amigos & 0,72 & 1 & 0,395 & 0,75 & {$[0,431-1,317]$} \\
\hline
\end{tabular}

Los valores p que están señalados con un asterisco indica las subescalas estadísticamente significativas. 


\section{Factores familiares de los estudiantes que consumen sustancias psicoactivas}

De los estudiantes que respondieron que han consumido alguna vez en su vida el $46,5 \%$ viven en una familia extendida y el $26,7 \%$ en una familia nuclear. Se comprobó que no existe una relación significativa entre el tipo de familia y el consumo de sustancias psicoactiva de los estudiantes $\left(x^{2}=7,140\right.$, $p=0,308)$.

Con respecto al nivel educativo de los padres el 44,2\% de los escolares respondió que su madre había cursado hasta secundaria y en un $17,4 \%$ hasta primaria. Se comprobó que no existe una relación significativa entre el grado de escolaridad de la madre y el consumo de sustancias psicoactiva de los estudiantes $\left(x^{2}=9,159, p=0,241\right)$. Con respecto al padre un $22,1 \%$ sus estudios son solo hasta primaria y un $29,1 \%$ estudio hasta primaria, tampoco se evidenciaron diferencias significativas $\left(x^{2}=4,014\right.$, $p=0,547$ ).

Cabe resaltar que la edad promedio de consumo de sustancia psicoactivas en los estudiantes es de $15,26 \pm 1,35$ años.

\section{Discusión}

Las acciones de prevención en el marco de la política nacional para la reducción del consumo de sustancias psicoactivas y su impacto, tienen algún efecto en la disminución del consumo en la región ya que según el estudio del año 2016 en escolares colombianos, el $70,7 \%$ de ellos usaron una sustancia psicoactiva alguna vez en la vida(10), cifra mayor a lo encontrado en el presente estudio, donde el $36,6 \%$ de los escolares encuestados refirieron haberlas usado, información que es equivalente a lo encontrado en una Institución educativa pública del municipio de Jamundí Valle, Colombia donde el 35\% de 146 estudiantes encuestados afirmaron haber consumido alguna de las sustancias(23).

Se encontró en los adolescentes que afirmaron haber consumido sustancias psicoactivas alguna vez en la vida, que el 16,2\% de ellos consumió alcohol, el 0,9\% marihuana, el $0,4 \%$ tabaco, el $0,4 \%$ estimulantes, lo cual coincide en gran medida con lo encontrado en el estudio nacional de consumo de sustancias psicoactivas para el año 2016, donde el alcohol sigue siendo la sustancia psicoactiva más usada en los adolescentes ${ }^{(10)}$ y en los universitarios de acuerdo a lo encontrado en 2015 en la ciudad de Pasto (Colombia)(26). Estos datos evidencian la permisividad social y el poco cumplimiento de la legislación colombiana en lo relacionado al alcohol, dado que se establece que la venta de licor está prohibida para personas menores de 18 años ${ }^{(4)}$.

Al considerar el grado escolar de los estudiantes donde se presenta mayor consumo, cuando se considera el conjunto completo de sustancias, en Colombia se observó un aumento estadísticamente significativo en los casos existentes desde un $42 \%$ en los escolares de séptimo grado, hasta un $77 \%$ en los de undécimo grado(10), información ratificada por el presente estudio; a medida que va aumentando el grado escolar, la prevalencia del consumo se acrecienta.

Por lo anterior, es indispensable diseñar estrategias educativas que controlen el consumo de este tipo de sustancias en los escolares, ya que las escuelas son plataformas importantes en la prevención del uso de sustancias y la promoción de la salud de la comunidad, teniendo en cuenta que los adolescentes pasan una gran cantidad de tiempo dentro y alrededor de estas, donde se ven directa e indirectamente afectados por las normas y la cultura de la escuela y la comunidad, además del aspecto educativo de la Institución.

En lo relacionado con los lugares donde se presenta el consumo de sustancias psicoactivas, un estudio realizado en El Espinal Tolima (Colombia) publicado en 2017, encontró que un alto porcentaje de adolescentes consume estas sustancias en la casa de sus amigos y fiestas, seguido por su propia casa y el colegio(28), situación similar a la observada en el presente estudio donde se evidencia que el lugar de menor consumo es el colegio y sus alrededores.

La familia es un pilar fundamental en el acompañamiento permanente del adolecentes, no solo en los entornos sociales, sino dentro del hogar ya que los jóvenes no solo consumen este tipo de sustancias en sitios donde es fácil el acceso, sino que pueden hacerlo en su vivienda sin el debido acompañamiento familiar, de ahí la importancia de que los padres se involucren en el entorno de los adolescentes(10,12,17-18). Investigaciones recientes afirman que vivir con ambos padres entendida esta como familia nuclear, tiene un efecto protector mayor frente al consumo de sustancias psicoactivas, que en 
los casos donde los adolescentes viven con los padres por separado o con familia ensamblada(12,13). Sin embargo, existe estudios donde no han encontrado diferencias estadísticamente significativas en el índice de consumo de sustancias psicoactivas según el tipo de familia(14), situación similar a la presentada en este estudio; lo que indica que más allá de la convivencia con uno o ambos padres, el tiempo de dedicación hacia los hijos, es uno de los factores que podría incidir en el consumo de este tipo de sustancias.

Teniendo en cuenta que la presente investigación evidenció que el acompañamiento o involucramiento de los padres con sus hijos, son un factor protector frente al consumo de sustancias psicoactivas $(p<0,05$; $O R<1$ ), se confirma que los adolescentes que cuentan con padres o adultos cuidadores involucrados en sus vidas, presentan menos niveles de prevalencias de consumo de sustancias tanto licitas como ilícitas, datos ratificados en otras investigaciones $(15,17)$. Adecuados procesos de supervisión y constantes muestras de afecto, genera en los adolescentes el desarrollo de un mayor autocuidado, desencadenando en ellos actitudes responsables, con mayor autoestima y autocontrol ante la influencia de los pares, que durante la adolescencia puede ser decisiva en la adopción de conductas de riesgo(10,12,18).

En una investigación realizada en 2012 en México evidenciaron que existía mayor prevalencia de uso de marihuana, tranquilizantes y cocaína en los adolescentes cuyos padres tenían una escolaridad de secundaria o más(16), sin embargo en la presente investigación se encontró que la escolaridad de los padres no influye en el consumo de sustancias psicoactivas de los jóvenes, ratificando que factores como el tipo de familia, nivel socioeconómico y escolaridad de padres, no constituyen un factor decisivo a la hora del consumo de sustancias psicoactiva.

Con relación a la influencia que tienen los amigos en el consumo de sustancias psicoactivas, se encontró que los escolares tienen 6,5 veces más posibilidades de consumir sustancias psicoactivas cuando se relacionan con amigos que consumen [OR=6,555 IC95\% (3,147-13,653)], similar a lo encontrado en una investigación publicada en 2013(21) donde evidenciaron que durante la etapa de la adolescencia el grupo de amigos pasan a ser una de las áreas más importantes del joven y el formar parte de un grupo y ser aceptado en éste, se convierte en una prioridad, lo cual beneficia la socialización e independencia, pero puede ser causa de riesgo si el grupo influye para que se realicen conductas que ponen en riesgo la salud del adolescente.

Se observó que los escolares que tienen familiares que consumen, tienen 2,2 veces más posibilidades de consumir sustancias psicoactivas, frente a los estudiantes que cuentan con un círculo familiar donde no consumen, [OR=2,231 IC95\% (1,292$3,852)]$, apoyando lo presentado por López et al.(20) quienes determinaron que el no uso de drogas entre familiares de primer grado, se considera un factor interpersonal importante que cohíbe el uso de drogas cuando se les presenta la oportunidad a los adolescentes.

Esta investigación contribuye a la estadística nacional y del departamento de Risaralda, en cuanto al conocimiento sobre el consumo de sustancias psicoactivas en adolescentes de la ciudad de Pereira; después de realizar una búsqueda sistemática de la literatura no se ha descrito en la ciudad la prevalencia de consumo de sustancias psicoactivas y los factores psicosociales asociados en adolescentes de la ciudad entre los 10 y 19 años. Por este motivo, se considera un aporte significativo en la ciencia de gran impacto social en nuestra región, que permite la generación de posibles medidas de atención en salud pública de acuerdo al riesgo. De igual forma permite hacer una evaluación de las medidas implementadas en la región para la prevención del consumo en población de tan alto riesgo.

\section{Conclusiones}

El consumo de sustancias psicoactivas en la población escolar participante, muestra una elevada prevalencia; los adecuados niveles de supervisión de los padres en la vida de los adolescentes son un factor protector frente al consumo de sustancias psicoactivas; se recomienda avanzar en la educación para enfrentarlas, proporcionando herramientas a las familias, maestros y escolares, para abordar esta problemática, sin daños por la experimentación natural a esta edad. Es importante que las instituciones educativas identifiquen la necesidad de elaborar estrategias que fomenten la unión familiar, hábitos de vida saludables y que incluyan la percepción de los riesgos y utilización del tiempo libre. 
Conflicto de intereses: Los autores no presentan conflictos de interés.

\section{Referencias}

1. Organización Mundial de la Salud. Adolescentes: riesgos para la salud y soluciones [Internet]. 2017. [cited 2020 Mar 13] Available from: http://www.who.int/mediacentre/factsheets/fs345/es/\#c ontent

2. Gaete V. Desarrollo psicosocial del adolescente. Rev Chil Pediatr [Internet]. 2015;86(6):436-43. Available from: https://scielo.conicyt.cl/scielo.php?pid=S037041062015000600010\&script=sci_arttext\&tlng=en

3. ONU. Asamblea General Consejo Económico y Social. Informe sobre la juventud mundial [Internet]. 2005. Available from: https://undocs.org/pdf?symbol=es/A/60/61

4. Ministerio de Salud y Protección Social. Plan Nacional para la promoción y la atención del consumo de sustancias psicoactivas 2014 - 2021 [Internet]. 2017 [cited 2020 Mar 13]. Available from: https://www.minsalud.gov.co/sites/rid/Lists/BibliotecaDi gital/RIDE/VS/PP/plan-nacional-consumo-alcohol-20142021.pdf

5. Ministerio de Justicia y del Derecho y el Observatorio de Drogas de Colombia(ODC). Reporte de drogas de Colombia [Internet]. Bogotá (Colombia): Minjusticia; 2017 [cited 2020 Mar 13]. Available from: http://www.odc.gov.co/Portals/1/publicaciones/pdf/odclibro-blanco/reporte_drogas_colombia_2017.pdf

6. Congreso de Colombia. Ley 124 de 1994 Prohibición del expendio de bebidas embriagantes a menores de edad. 1994. (Diario Oficial No. 41230 de febrero 18 de 1994, Bogota, Colombia). Disponible en: http://www.secretariasenado.gov.co/senado/basedoc/ley_ 0124_1994.html

7. Congreso de Colombia. Ley 1335 de 2009 Disposiciones sobre la venta de productos de tabaco a menores de edad. 2009. (Diario Oficial No. 47417 de julio 21 de 2009, Bogota, Colombia). Disponible en: http://www.secretariasenado.gov.co/senado/basedoc/ley_ 1335_2009.html

8. Ministerio de Protección Social, Republica de Colombia. Encuesta Nacional sobre Consumo de Sustancias Psicoactivas en Jóvenes Escolarizados de 12 a 17 Años en Colombia en el 2004 [Internet]. 2005. Available from: https://www.minsalud.gov.co/sites/rid/Lists/BibliotecaDi gital/RIDE/VS/ED/GCFI/Base\%20de\%20datos\%20Consum o\%20SPA\%20\%20escolares\%202004\%20informe.pdf

9. Observarorio de Drogas de Colombia, Ministerio de Educación Nacional, Ministerio de Salud y Protección Social. Estudio nacional de consumo de sustancias psicoactivas en poblacion escolar colombia - 2011 [Internet]. 2013. Available from:

https://www.unodc.org/documents/colombia/Documento stecnicos/Estudio_Consumo_Escolares.pdf

10. Observatorio de Drogas de Colombia, Ministerio de Justicia y del Derecho, Ministerio de Educación Nacional, Ministerio de Salud y Protección Social, Comisión Interamericana para el Control del Abuso de Drogas (CICAD) de la Organización de los Estados Americanos (OEA). Estudio Nacional de consumo de sustancias psicoactivas en población escolar Colombia 2016 [Internet]. Observatorio de drogas de Colombia. 2016. Available from: http://www.odc.gov.co/Portals/1/publicaciones/pdf/cons umo/estudios/nacionales/CO03142016_estudio_consumo_ escolares_2016.pdf

11. Restrepo M. Consumo de sustancias psicoactivas y migración, en entornos escolares, en cinco municipios del eje cafetero [Tesis de grado maestria] [Internet]. Universidad Tecnológica de Pereira; 2015 [cited 2018 Jun 29]. Available from: http://hdl.handle.net/11059/5701

12. Recabarren V. Involucramiento parental y consumo de drogas en escolares de Chile [Internet]. Vol. 19, Observatorio chileno de Drogas. 2015. Available from: https://www.senda.gob.cl/wpcontent/uploads/boletines/Boletin\%2019\%20Involucrami ento $\% 20$ parental $\% 20 \mathrm{y} \% 20$ consumo $\% 20 \mathrm{de} \% 20$ drogas $\% 2$ 0en\%20escolares\%20de\%20Chile.pdf

13. Jiménez A, Castillo K, Cisneros M, Salazar M. Incidencia de la disfuncionalidad familiar en el consumo de drogas en jóvenes del reparto Rubén Darío, León Sureste [Monografía] Universidad Nacional Autonoma de Nicaragua [Internet]. 2013. Available from: http://riul.unanleon.edu.ni:8080/jspui/bitstream/1234567 89/5808/1/225324.pdf

14. Moreno N, Palomar J. Factores familiares y psicosociales asociados al consumo de drogras en adolscentes. Rev Interam Psicol [Internet]. 2017;51(2):141-51. Available from:

https://www.redalyc.org/articulo.oa?id=28454546001

15. Molero M, Pérez M, Gázquez J, Barragán A. Análisis y perfiles del consumo de drogas en adolescentes: percepción del apoyo familiar y valoración de consecuencias. Aten Fam [Internet]. 2017;24(2):56-61. doi: https://doi.org/10.1016/j.af.2017.02.001

16. Villatoro J, Mendoza M, Moreno M, Oliva N, Fregoso D, Bustos M, Fleiz C, Mujica R, López M, Medina M. Tendencias del uso de drogas en la Ciudad de México: Encuesta de Estudiantes del 2012. Salud Ment [Internet]. 2014;37(5):423-35. Available http://www.scielo.org.mx/pdf/sm/v37n5/v37n5a9.pdf

17. Valenzuela E. Padres involucrados y uso de drogas: un análisis empírico. Estud públicos [Internet]. 2006;101:14764. Available from: http://dialnet.unirioja.es/servlet/articulo?codigo=1994138 \&info=resumen\&idioma $=$ SPA

18. Méndez J. Consumo de drogas e involucramiento parental entre estudiantes de secundaria de Costa Rica durante el 2015. Drugs Addict Behav [Internet]. 2017;2(2):193-205. Available from: http://www.funlam.edu.co/revistas/index.php/DAB/article /view/2440

19. Lopez C, Neumark Y. Prevalence and determinants of resistance to use drugs among adolescents who had an opportunity to use drugs. Drug Alcohol Depend [Internet]. 2015;149:55-62. doi:10.1016/j.drugalcdep.2015.01.015

20. Rossow I, Keating P, Felix L, Mccambridge J. Does parental drinking influence children' s drinking ? A systematic review of prospective cohort studies. Addiction [Internet]. 2015;111:204-17. doi:10.1111/add.13097

21. Sánchez C, Andrade P, Betancourt D, Vital G. Escala de Resistencia a la Presión de los Amigos para el Consumo de Alcohol. Acta Investig Psicológica (Psychological Res Rec [Internet]. 2013;3(1):917-29. Doi: 10.1016/S2007- 
4719(13)70942-6

22. Rodriguez M, Perozo C, Matute J. Resistencia de la presión de grupo y consumo de alcohol en adolescentes. Rev Psicol Univ Antioquia [Internet]. 2014;6(1):25-40. Available from: https://dialnet.unirioja.es/descarga/articulo/4947507.pdf

23. Espinosa K, Hernández M, Cassiani C, Cubides Á, Martínez M. Factores relacionados con el consumo de sustancias psicoactivas en una institución educativa de Jamundí Valle, Colombia. Rev Colomb Psiquiatr [Internet]. 2016;45(1):2-7. doi: https://doi.org/10.1016/j.rcp.2015.06.001

24. Revelo M, Revelo A, Velasco R. Factores de riesgo asociados al consumo de Sustacias psicoactivas en jovenes de 8 a 21 años. Ipiales, Nariño 2000. Univ Salud. [Internet]. 2002;1(3):55-9. Available from: https://revistas.udenar.edu.co/index.php/usalud/article/vi ew/322

25. Zarrouq B, Bendaou B, El Asri A, Achour S, Rammouz I, Aalouane R, et al. Psychoactive substances use and associated factors among middle and high school students in the North Center of Morocco: A cross-sectional questionnaire survey. BMC Public Health [Internet]. 2016;16(486):1-9. Available from: http://dx.doi.org/10.1186/s12889-0163143-5

26. Betancourth S, Tacán L, Cordoba E. Consumo de alcohol en estudiantes universitarios colombianos. Rev Univ y Salud [Internet]. 2017;19(1):37-50. Available from: http://revistas.udenar.edu.co/index.php/usalud/article/vie $\mathrm{w} / 2861 / \mathrm{pdf}$

27. Roderick E, Penney J, Murrells T, Dargan P, Norman I. Epidemiology of adolescent substance use in Norfolk schools. Qjm. 2018;111(10):699-706. doi: https://doi.org/10.1093/qjmed/hcy153

28. Aguirre N, Aldana O, Bonilla C. Factores familiares de riesgo de consumo de sustancias psicoactivas en estudiantes de una institución de educación media técnica de Colombia. Rev Salud Pública [Internet]. 2017;19(1):3-9. Available from: http://revistas.unal.edu.co/index.php/revsaludpublica/arti cle/view/41785

29. Segunda Conferencia Mundial sobre la Integralidad de la Investigación. Declaración de Singapur sobre la intregridad en la investigación [Internet]. 2010 p. 1-2. Available from: https://www.conicyt.cl/fondap/files/2014/12/DECLARACI ÓN-SINGAPUR.pdf

30. Ministerio de Salud. Resolución 8430 de 1993 Normas científicas, técnicas y administrativas para la investigación en salud. [Internet]. Ministerio De Salud 1993 p. 1-19. Bogota, Colombia Available from: https://www.minsalud.gov.co/sites/rid/Lists/BibliotecaDi gital/RIDE/DE/DIJ/RESOLUCION-8430-DE-1993.PDF

31. Organización Internacional de Estandarización. ISO 26000 visión general del proyecto [Internet]. 2010 [cited 2020 Mar 13]. Available from: https://www.iso.org/files/live/sites/isoorg/files/archive/ pdf/en/iso_26000_project_overview-es.pdf 